\title{
Cole Parameter Estimation from the Modulus of the Electrical Bioimpeadance for Assessment of Body Composition. A Full Spectroscopy Approach.
}

\author{
R. Buendia ${ }^{1,2,3}$, R. Gil-Pita ${ }^{2}$ and F. Seoane ${ }^{1,3}$ \\ 1. School of Engineering, University of Borås, SE-501 90 Borås, SWEDEN \\ 2. Department of Signal Theory and Communications, University of Alcala, ES-28871, Madrid, SPAIN \\ 3. School of Technology and Health, Royal Institute of Technology, SE-141 52, Huddinge, SWEDEN \\ 4. E-mail ruben.buendia@hb.se
}

\begin{abstract}
Activities around applications of Electrical Bioimpedance Spectroscopy (EBIS) have proliferated in the past decade significantly. Most of these activities have been focused in the analysis of the EBIS measurements, which eventually might enable novel applications. In Body Composition Assessment (BCA), the most common analysis approach currently used in EBIS is based on the Cole function, which most often requires curve fitting. One of the most implemented approaches for obtaining the Cole parameters is performed in the impedance plane through the geometrical properties that the Cole function exhibit in such domain as depressed semi-circle. To fit the measured impedance data to a semi-circle in the impedance plane, obtaining the Cole parameters in an indirect and sequential manner has several drawbacks. Applying a Non-Linear Least Square (NLLS) iterative fitting on the spectroscopy measurement, obtains the Cole parameters considering the frequency information contained in the measurement. In this work, from experimental total right side EBIS measurements, the BCA parameters have been obtained to assess the amount and distribution of whole body fluids. The values for the BCA parameters have been obtained using values for the Cole parameters estimated with both approaches: circular fitting on the impedance plane and NLLS impedance-only fitting. The comparison of the values obtained for the BCA parameters with both methods confirms that the NLLS impedance-only is an effective alternative as Cole parameter estimation method in BCA from EBIS measurements. Using the modulus of the Cole function as the model for the fitting would eliminate the need for performing phase detection in the acquisition process, simplifying the hardware specifications of the measurement instrumentation when implementing a bioimpedance spectrometer.
\end{abstract}

Keywords: Bioimpedance, spectroscopy measurements, Cole Analysis, Body Composition

\section{Introduction}

Nowadays, measuring the Electrical Bioimpedance (EBI) in humans is a common practice in several clinical applications e.g. electronic biopsy for skin cancer screening [1], Body Composition Analysis (BCA) for assessment on body fluids distribution [2], impedance cardiography for non-invasive hemodynamic monitoring [3]. Several EBI applications make use of EBI spectroscopy (EBIS) measurements analyzing the impedance spectrum and, in most cases, a Cole-based model analysis is performed.

$$
Z_{\text {Cole }}(\omega)=R_{\infty}+\frac{R_{0}-R_{\infty}}{1+(j \omega \tau)^{\alpha}}
$$

To perform a Cole model analysis, EBIS data must be fitted to the Cole Function, see eq. (1), [4]. The Cole function experimentally resembles EBIS data from a single dispersion in the given frequency range and it is defined by four parameters $R_{0}, R_{\infty}, \alpha$ and $\tau$. The Cole parameters can be used for visualization producing the Cole plot and as data features to characterize an EBI system. Through further processing they can be also used for tissue constitution assessment like it is the case of BCA applications [5].

In BCA, using the values obtained from EBIS data for the Cole parameters and applying Hanai mixture theory [6], [7] and [2] or empirically derived prediction equations [8], it is possible to estimate the value of the BCA parameters: TBW, ECF, ICF and FM.

The estimation of the values of the Cole parameters from the EBIS data is usually obtained through iterative Curve fitting [9], [10], [11]. Since the impedance is a complex function of frequency, curve fitting can be done on the spectral domain or in the impedance plane [9] [12] and [13].

The impedance-only approach presented in [13] estimates the value of the Cole parameters from the modulus of the EBI, allowing the use of a non-phase sensitive spectrometer. This reduction of hardware requirements is obtained at the expenses of producing the parameter estimation on the impedance plane disregarding the frequency information.

Recently, the Non-linear Least Squares (NLLS) approach to fit EBIS measurements into the Cole function on the frequency domain was theoretically introduced by Ayllon et al in [14] and empirically proven by Buendia in [15]. Although in both works the NLLS approach was applied on the immitance spectral components of the complex EBI data, which require EBI measurements taken with a phase sensitive EBI spectrometer, the NLLS approach allows performing Cole parameter estimation on the modulus of the impedance as well.

Combining both the impedance-only estimation approach suggested by Ward et al in [13] and the NLLS method, it would be possible to implement a Cole function 
fitting method that would estimate the Cole parameter in the frequency domain and would not require phasesensitive EBI measurements. A recent algorithm evaluation performed by Nordbotten et al. in [16] confirms the validity of such approach.

To validate the use of the NLLS impedance-only approach for a well known and spread application of EBIS, in this work, the BCA parameters have been estimated through Cole function fitting from total right side wrist-toankle EBI measurements. The BCA parameters have been calculated with the Bioimp software for assessment of body composition while, for comparison purposes, the Cole function fitting has been performed both, applying the NLLS method on the spectrum of the impedance modulus and using the Cole fitting tool available on Bioimp software.

A positive validation of the NLLS impedance-only approach would enable the design of impedance spectrometer for body composition analysis and assessment on nutritional status without the need of phase detection capabilities, which would reduce considerably the complexity of the hardware requirements.

\section{Materials and methods}

\section{A. Cole Based analysis and BCA parameters estimation}

In 1940 Kenneth S. Cole introduced the Cole equation, eq (1), an empirical complex nonlinear function of frequency that accurately fitted experimental EBI measurements. Such function is built by 4 parameters $R_{0}$, $R_{\infty}, \alpha$ and $\tau$, but only 2 of them, the resistance at DC frequency $R_{0}$ and the resistance at infinite frequency $R_{\infty}$, are used to estimate the BCA parameters through Hanai's mixture theory [6]. Using the Cole parameters, together with morphological data from a human subject and certain constants [17], it is possible to predict the volume of the extra- and intracellular fluid and consequently the total content of body water, ECF, ICF and TBW correspondently, which are known as BCA parameters. Such parameters are estimated according to equations 2 and $3,[17] \&[18]$.

$$
E C F=\frac{1}{100} \sqrt[3]{\frac{K_{b}^{2} \rho_{e c f}^{2}}{D_{b}} * \frac{H^{4} W}{R_{0}^{2}}}
$$

The ECF parameter in liters is obtained with eq. (2), Where $W$ is body weight in $\mathrm{kg}, H$ is height in $\mathrm{cm}, R_{0}$ is the value of the Cole parameter in $\Omega, K_{b}$ is the body proportion, typically 4.3 for wrist to ankle measurements, $\rho_{e c f}$ is the resistivity of the extracellular fluid and $D_{b}$ is the body density in $\mathrm{kg} / \mathrm{l}$ with a estimated value of 1.05 .

Once the value ECF is estimated introducing the value for $R_{0}$, the ICF parameter is calculated following eq. (4). Note that equations (2), (3) and (4) have been adapted to the Cole parameters and the nomenclature of body fluid and not body water.

$$
\left(1+\frac{I C F}{E C F}\right)^{5 / 2}=\left(\frac{R_{0}}{R_{\infty}}\right)\left(1+\frac{\rho_{i}}{\rho_{e}} \frac{I C F}{E C F}\right)
$$

Where ICF and ECF are the volumes of intra and extra cellular fluid in liters respectively and $\rho_{i}$ and $\rho_{e}$ are the apparent intra- and extracellular resistivities respectively [17].

Eq. (3) can be solved by expanding it into the form of eq. (4) where $x=I C F / E C F$.

$$
\begin{aligned}
x^{5}+5 x^{4}+(10 & \left.-\left(\frac{R_{0}}{R_{\infty}}\right)^{2}+\left(\frac{\rho_{i}}{\rho_{e}}\right)^{2}\right) x^{2} \\
& +\left(5-2\left(\frac{R_{0}}{R_{\infty}}\right)^{2}+\left(\frac{\rho_{i}}{\rho_{e}}\right)\right) x+1-\left(\frac{R_{0}}{R_{\infty}}\right)^{2}=0
\end{aligned}
$$

The expression in eq. (4) can be solved iteratively by using various values of $x$ between 0 and 5 , until the result is approximately zero (within 0.00001).

Then ICF may be calculated from $x$ and ECF (obtained earlier) as in eq. (5) [17].

$$
I C F=x * E C F
$$

Once the values of ECF and ICF are obtained, the value of the TBW is consequently obtained just by addition of ICW and ECW like in eq. (6).

$$
T B W=E C W+I C W
$$

The amount of Fat Free Mass (FFM) can be derived directly from the TBW value applying the hydration constant, $K_{h}$, as in eq. (7). The typical value for $K_{h}$ is 0.732 [19].

$$
F F M=\frac{T B W}{K_{h}}
$$

Therefore to work out the value of the fat mass (FM) that is the parameter used for analisys eq. (8) is used.

$$
F M=W-F F M
$$

In this work the tool used to obtain the BCA parameters from the Cole parameters has been the Bioimp software analysis tool for Body composition assessment (v5.3.1.1, Impedimed Ltd, Brisbane).

\section{B. Non-Linear Least Squares for Cole Function Fitting}

This method aims to obtain the best coefficients for a given model that fits the curve, the method given by eq. (9) aims to minimize the summed squared of the error between the measured data value and the fitted value $\overline{\left|Z_{i}\right|}$, which is

$$
\min \sum_{i=1}^{N} e_{i}^{2}=\min \sum_{i=1}^{N}\left(\left|Z_{i}\right|-\overline{\left|Z_{i}\right|}\right)^{2}
$$




$$
\overline{\left|Z_{i}\right|}=\sqrt{\left(R_{\infty}+\frac{\left(R_{0}-R_{\infty}\right)\left(1+(\omega \tau)^{\alpha} \cos (\alpha \pi / 2)\right)}{1+2(\omega \tau)^{\alpha} \cos (\alpha \pi / 2)+(\omega \tau)^{2 \alpha}}\right)^{2}+\left(\frac{\left(R_{0}-R_{\infty}\right)(\omega \tau)^{\alpha} \sin (\alpha \pi / 2)}{1+2(\omega \tau)^{\alpha} \cos (\alpha \pi / 2)+(\omega \tau)^{2 \alpha}}\right)^{2}}
$$

obtained from the modulus of the Cole function shown in eq. (10).

Where $\mathrm{N}$ is the number of frequency data points included in the fitting. This approach was validated in [14] as working approach to estimate the Cole parameters from the resistance spectrum and the reactance spectrum, as well as from the complex impedance spectrum. In this case, the minimization cost function has been built with the modulus of the complex EBI. Thus the term $\left|Z_{i}\right|$ is the modulus of the measured impedance and $\overline{\left|Z_{i}\right|}$ is the absolute value of the Cole function in eq. (1) as shown in (10).

This method has been implemented in Matlab, fitting the generated data to a non-linear real parametric model with coefficients, using the natural frequency $\omega$ as an independent variable. Performing the curve fitting using a Cole-based function like in eq. (1) allows the estimation of the values for the four Cole parameters.

\section{EBI Measurements and Descriptive Statistics}

Right side 4-electrode wrist-to-ankle EBI spectroscopy measurements have been taken in five healthy volunteers. The EBIS measurements were performed with the SFB7 bioimpedance spectrometer manufactured by Impedimed ltd. using repositionable Red Dot $\mathrm{Ag} / \mathrm{AgCl}$ electrodes manufactured by $3 \mathrm{M}$. The frequency range of performed EBI measurements was 3.096 to $1000 \mathrm{kHz}$ and 100 complex EBI spectroscopy measurements were obtained for each of the volunteers.

The body parameters of the volunteers can be observed in Table I. Subject 5 is female and all the others are male.

Table I. Subjects body features

\begin{tabular}{cccc}
$\begin{array}{c}\text { Subjects } \\
\text { Features }\end{array}$ & $\begin{array}{c}\text { Age } \\
(\text { years })\end{array}$ & $\begin{array}{c}\text { Height } \\
(\mathrm{cm})\end{array}$ & $\begin{array}{c}\text { Weight } \\
(\mathrm{kg})\end{array}$ \\
\hline Subject 1 & 32 & 176 & 99.0 \\
Subject 2 & 30 & 165 & 60.0 \\
Subject 3 & 26 & 174 & 94.4 \\
Subject 4 & 26 & 182 & 83.8 \\
Subject 5 & 24 & 175 & 72.5
\end{tabular}

Table II. Proportionality and body resistivity constants used with Bioimp

\begin{tabular}{ccc} 
& Male & Female \\
\hline$\rho_{e}$ & 340 & 322 \\
$\rho_{i}$ & 859 & 784 \\
Body density $\left(D_{b}\right)$ & & \multicolumn{2}{c}{1.05} \\
Body proportion $\left(K_{b}\right)$ & \multicolumn{2}{c}{4.30} \\
Hydration constant $\left(K_{h}\right)$ & \multicolumn{2}{c}{0.732}
\end{tabular}

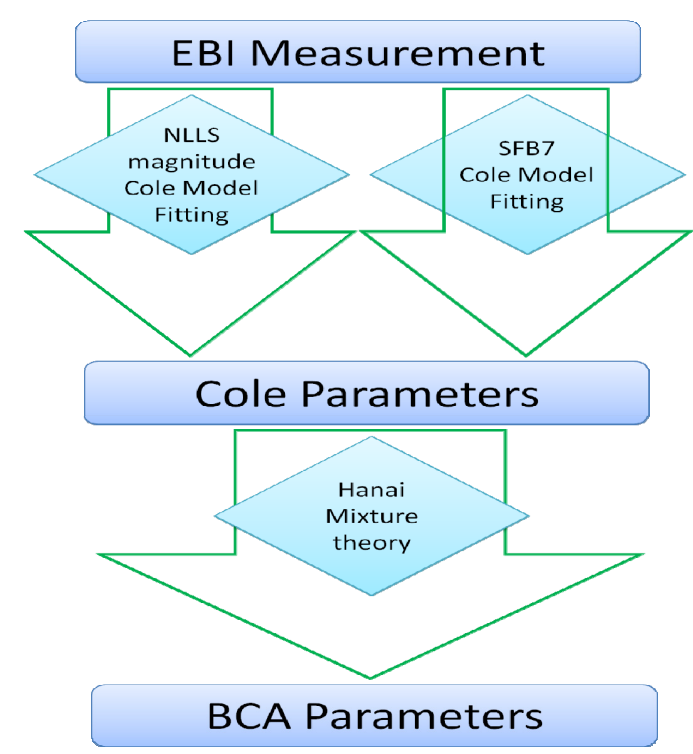

Fig. 1: Work Flow

\section{EBI Data Analysis and Comparison}

As the work flow on Fig. 1 indicates, the Cole curve fitting and BCA parameters estimation were performed on a total of 500 measurements. The mean, minimum and maximum values of the BCA parameters estimated from the corresponding Cole parameters were calculated for each of the subjects. The values obtained for both Cole curve fittings were compared.

The performed EBI measurements were fitted to the Cole function with both, the curve fitting implemented on the Bioimp software and the NLLS approach on the modulus of the impedance implemented with MATLAB.

The Cole fitting with the Bioimp software was produced with the following curve fitting setup, $\mathrm{Td}$ compensation off, rejection threshold of $1 \%$ and frequency limits from $3.096 \mathrm{kHz}$ to $1000 \mathrm{kHz}$, i.e. full spectral range.

Two sets of BCA parameters were obtained per subject, each of them corresponding to the Cole parameters estimated with each of the approaches. The BCA parameters were calculated using the BCA tool of Bioimp with the morphological subject information listed in Table I and the proportionality and body resistivity constants indicated in Table II.

\section{Results}

The following figures and tables present the values of the BCA parameters obtained with both fitting processes from the EBIS measurement for all 5 subjects.

Table III reports the mean values for the BCA parameters in liters and kilograms correspondingly to the fluid body contents and the fat mass. 
Table III. Mean values for the BCA parameters of each subject

\section{Subject 1}

Subject 2

Subject 3

Subject 4

Subject 5

\begin{tabular}{ccccccccccc}
\hline & NLLS & \multirow{2}{*}{ Bioimp } & NLLS Z & Bioimp & NLLS Z & Bioimp & NLLSZ & Bioimp & NLLSZ & Bioimp \\
\hline TBW (1) & 56.3 & 56.4 & 32.6 & 33.0 & 49.6 & 49.7 & 50.1 & 50.4 & 33.2 & 33.4 \\
ECF (1) & 28.4 & 28.4 & 17.8 & 17.7 & 26.0 & 26.0 & 26.7 & 26.6 & 20.1 & 20.1 \\
ICF (1) & 27.9 & 28.0 & 14.8 & 15.3 & 23.6 & 23.7 & 23.4 & 23.8 & 13.1 & 13.4 \\
FM (kg) & 22.1 & 22.0 & 15.4 & 14.8 & 26.7 & 26.5 & 15.4 & 14.9 & 27.1 & 26.8
\end{tabular}

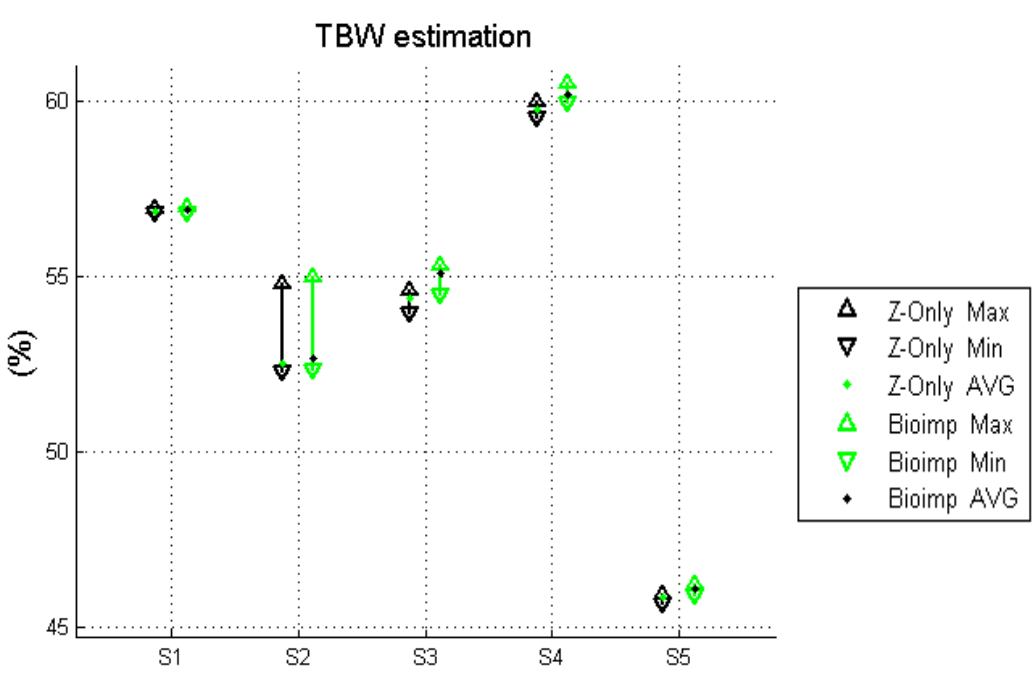

Fig. 2: Mean, minimum and maximum values for $\mathrm{TBW}$, in \%, estimated for all the subjects with both methods

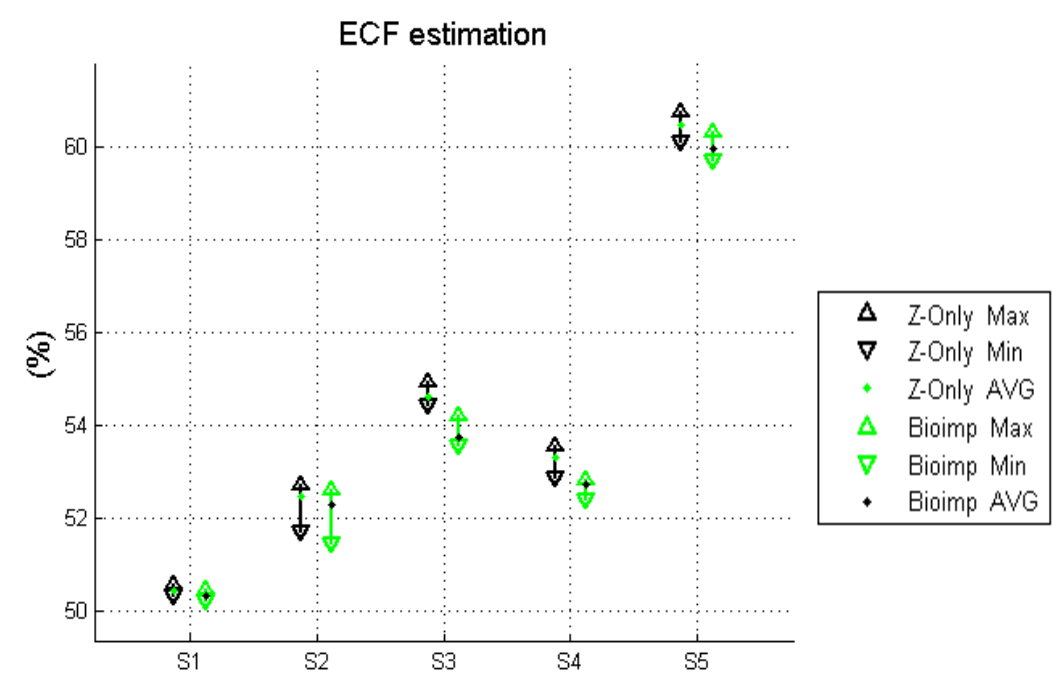

Fig. 3: Mean, minimum and maximum values for ECF, in \%, estimated for all the subjects with both methods

Figures 2-5 present the mean, minimum and maximum values, in \%, obtained for the BCA parameters, i.e. TBW, ECF, ICF or FM, per subject for both approaches, while Tables IV-VII contain the mean values and the standard deviation (SD).

Fig. 2 and Table IV present the amount of TBW in the body given as a percentage of the total body mass. A high
Table IV. Mean and Standard Deviation of the estimated values for TBW in $\%$

TBW

(\%)

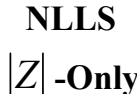

Bioimp

\begin{tabular}{lllll}
\cline { 2 - 5 } & Mean & SD & Mean & SD \\
\hline Subject 1 & 56.88 & 0.03 & 56.93 & 0.04 \\
Subject 2 & 54.40 & 0.12 & 55.11 & 0.16 \\
Subject 3 & 52.53 & 0.29 & 52.66 & 0.31 \\
Subject 4 & 59.78 & 0.08 & 60.19 & 0.08 \\
Subject 5 & 45.86 & 0.05 & 46.11 & 0.07
\end{tabular}

Table V. Mean and Standard Deviation of the estimated values for ECF in \%

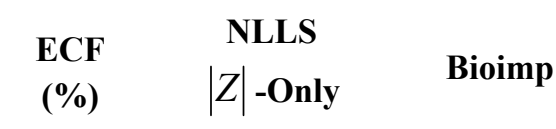

\begin{tabular}{lllll} 
& & & & \\
\cline { 2 - 5 } & Mean & SD & Mean & SD \\
\hline Subject 1 & 50.45 & 0.07 & 50.33 & 0.06 \\
Subject 2 & 54.62 & 0.08 & 53.75 & 0.12 \\
Subject 3 & 52.48 & 0.14 & 52.29 & 0.15 \\
Subject 4 & 53.31 & 0.10 & 52.72 & 0.08 \\
Subject 5 & 60.48 & 0.11 & 59.99 & 0.12
\end{tabular}




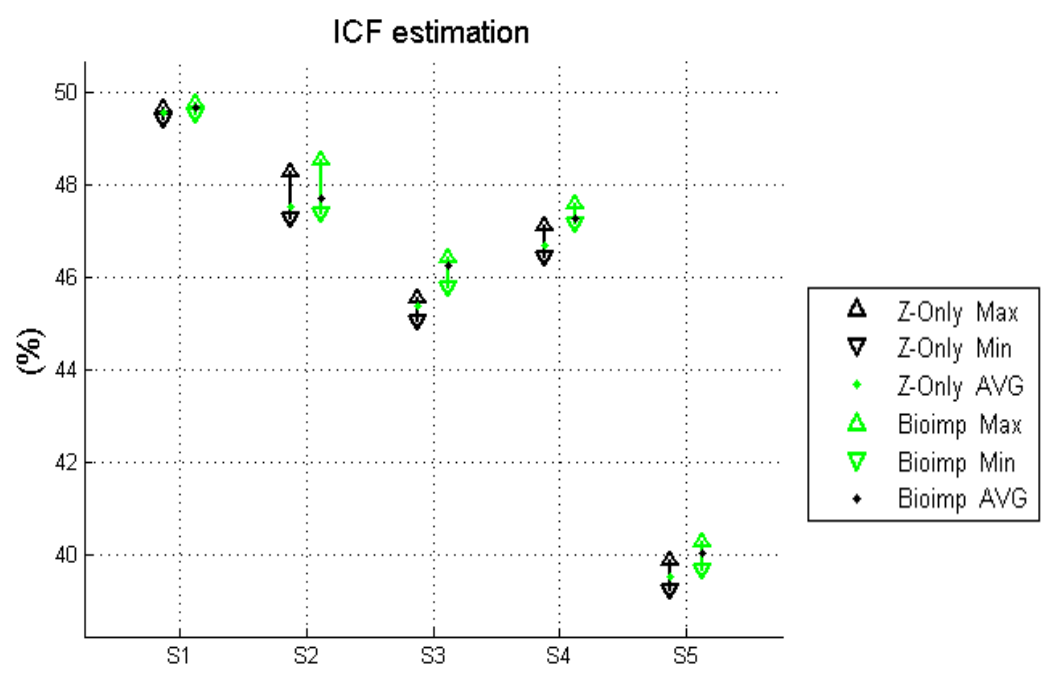

Fig. 4 Mean, minimum and maximum values for ICF, in \%, estimated for all the subjects with both methods

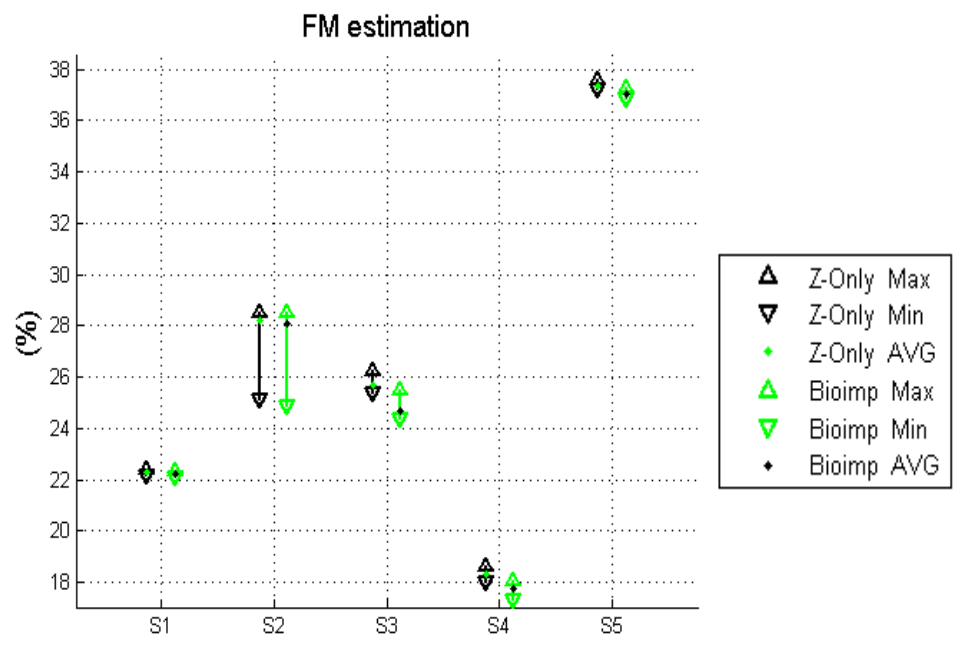

Fig. 5 Mean, minimum and maximum values for FM, in \%, estimated for all the subjects with both methods

of total body water. In this case the mean values estimated with the NLLS impedance-only fitting are slightly larger and the SD values are again slightly smaller, being nevertheless the result given by the two methods very close with a correlation coefficient of 0.9956 .

Fig. 4 and Table VI present the amount of fluid in the intracellular space given as a percentage of the amount of total body water. Since the values of ICF and EFC are
Table VI. Mean and Standard Deviation of the estimated values for ICF in $\%$

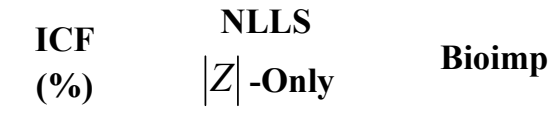

\begin{tabular}{lllll}
\cline { 2 - 5 } & Mean & SD & Mean & SD \\
\hline Subject 1 & 49.55 & 0.07 & 49.67 & 0.06 \\
Subject 2 & 45.38 & 0.08 & 46.25 & 0.12 \\
Subject 3 & 47.52 & 0.14 & 47.71 & 0.15 \\
Subject 4 & 46.69 & 0.10 & 47.28 & 0.08 \\
Subject 5 & 39.52 & 0.11 & 40.01 & 0.12
\end{tabular}

Table VII. Mean and Standard Deviation of the estimated values for FM in \%

FM

(\%)

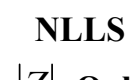

Bioimp

\begin{tabular}{lllll} 
& Mean & SD & Mean & SD \\
\hline Subject 1 & 22.30 & 0.04 & 22.23 & 0.05 \\
Subject 2 & 25.69 & 0.17 & 24.71 & 0.22 \\
Subject 3 & 28.24 & 0.40 & 28.06 & 0.43 \\
Subject 4 & 18.33 & 0.11 & 17.77 & 0.11 \\
Subject 5 & 37.35 & 0.07 & 37.01 & 0.09
\end{tabular}

Table VIII. Differences obtained on the estimation of the BCA parameters

\begin{tabular}{ccccc}
\multirow{2}{*}{$\Delta(\%) /(1)$} & \multicolumn{4}{c}{ (NLLS |Z|-Only) - Bioimp } \\
\cline { 2 - 5 } & TBW & ECF & ICF & FM \\
\hline Subject 1 & $-0.04 /-0.04$ & $0.12 / 0.04$ & $-0.12 /-0.09$ & $0.06 / 0.06$ \\
Subject 2 & $-0.71 /-0.42$ & $0.87 / 0.05$ & $-0.87 /-0.48$ & $0.97 / 0.59$ \\
Subject 3 & $-0.13 /-0.12$ & $0.19 / 0.03$ & $-0.19 /-0.15$ & $0.18 / 0.17$ \\
Subject 4 & $-0.41 /-0.35$ & $0.59 / 0.12$ & $-0.59 /-0.46$ & $0.57 / 0.47$ \\
Subject 5 & $-0.25 /-0.18$ & $0.49 / 0.05$ & $-0.49 /-0.24$ & $0.34 / 0.25$ \\
\hline Average & $-0.31 /-0.22$ & $0.45 / 0.06$ & $-0.45 /-0.28$ & $0.42 / 0.31$
\end{tabular}

Note: The percentage of ECF and ICF are referred to the TBW and the FM is expressed in percentage of the total mass and it is given in $\mathrm{kg}$ 
Table VIII presents the difference between the values obtained with the NLLS impedance-only method and the values produced from the Bioimp. fitting. From the values in Table VIII it is possible to observe that the difference on percentage of the estimated BCA parameter is below $1 \%$.

\section{Discussion}

The volume for the ECF obtained with both approaches is practically the same according to the obtained correlation coefficient. The volume differences observed on the estimation of the body fluid distribution occur mainly in the amount of ICF. The amount of volume estimated with the NLLS impedance-only fitting is slightly smaller than the amount of volume estimated by Bioimp. In any case a correlation coefficient of 0.9956, indicates that the difference obtained between both approaches is very small.

Since the only difference in the applied estimation process is the curve fitting method applied to obtain the Cole function and the Cole parameters, any difference in the obtained value for the BCA parameters comes from the curve fitting process. The negligible difference in the estimation of ECF indicates that the value estimated for $\mathrm{R}_{0}$ is very similar for both methods. This leaves the source for the difference obtained between both approaches in the estimation of the value $R_{\infty}$

When performing EBI measurements any capacitive parasitic effect associated to the measurement load or leads influence largely on the estimation of the reactance and the phase spectra, while the influence on the resistance or the modulus spectra is remarkable smaller [15]. If the estimation of the Cole parameters is done in the impedance plane [9], reactance vs. resistance, fitting the measured data to a semicircle, the reactance data containing corrupted values produced by capacitive leakage would lead to underestimation of the value of $R_{\infty}$, which consequently influences on the calculation of the value of ICF.

To compensate for such parasitic effect the Bioimp software has a built-in option known as Td compensation $[17,20]$. In this study such option has been left unused to allow for a fair comparison between fitting approaches, this way being able to show that the NLLS impedance-only fitting approach produces a slightly larger value for ICF distribution than the one obtained with the Bioimp fitting. In any case the measurements used in this study presented a reactance deviation at high frequencies that was completely un-noticeable to the naked eye, which indicates that the capacitive leakage present on the measurement was almost negligible [15].

The NLLS impedance-only not only presents the advantage of exhibiting a specific robustness to the effects of capacitive leakage due to the fact the modulus of the impedance spectrum is not affected by capacitive leakage as much as the phase or the reactance, but brings an additional and significant advantage also for the electronics of measurement acquisition systems. The measurement of the modulus of the impedance does not require phase detection, which decreases the complexity required to build the spectrometer device.

\section{Conclusion}

The performance exhibited by NLLS impedance-only approach for estimating the BCA parameters indicates that such fitting approach is a strong alternative to the currently in use Cole curve fitting-based on the impedance plane. In addition to the advantages related to the curve fitting and Cole parameters estimation processes, the use of the NLLS impedance-only allows the simplification of the measurement instrumentation eliminating the need for a phase detector. Consequently, since BCA and fluid distribution assessment is currently the application of electrical bioimpedance most spread worldwide, this novel Cole parameter estimation approach might have significant repercussions in the design of future electrical bioimpedance spectrometers.

\section{Acknowledgements}

The authors would like to acknowledge and show his appreciation to Brian Ziegelaar and Tim Essex from Impedimed ltd. for providing the necessary information about the processes implemented by the Bioimp software.

\section{References}

1. Aberg, P., et al., Skin cancer identification using multifrequency electrical impedance - A potential screening tool. IEEE Trans. Bio. Med. Eng., 2004. 51(12): p. 20972102. doi: 10.1109/TBME.2004.836523

2. Matthie, J.R., et al. Development of a commercial complex bio-impedance spectroscopic (CBIS) system for determining intracellular water (ICW) and extracellular water (ECW) volumes. in Proceedings of 8th International Conference on Electrical Bio-impedance. 1992. Kuopio, Finland, University of Kuopio.

3. Ferrario, C.M., et al., The role of noninvasive hemodynamic monitoring in the evaluation and treatment of hypertension. Ther Adv Cardiovasc Dis, 2007. 1(2): p. 113-8. doi: $10.1177 / 1753944707086095$

4. Cole, K.S., Permeability and impermeability of cell membranes for ions. Quant. Biol., 1940. 8: p. 110-122.

5. Moon, J.R., et al., Percent body fat estimations in college men using field and laboratory methods: a three-compartment model approach. Dyn Med, 2008. 7: p. 7. doi: $10.1186 / 1476-5918-7-7$

6. Hanai, T., Electrical properties of emulsions. Emulsion Science, London: Academic, 1968. 354-477.

7. Patel, R.V., et al., Estimation of total body and extracellular water using single- and multiple-frequency bioimpedance. Ann Pharmacother, 1994. 28(5): p. 565-9. 
8. Cornish, B.H., et al., Evaluation of multiple frequency bioelectrical impedance and Cole-Cole analysis for the assessment of body water volumes in healthy humans. Eur J Clin Nutr, 1996. 50(3): p. 159-64.

9. Cornish, B.H., B.J. Thomas, and L.C. Ward, Improved prediction of extracellular and total body water using impedance loci generated by multiple frequency bioelectrical impedance analysis. Phys. Med. Biol., 1993. 38(3): p. 337346. doi: 10.1088/0031-9155/38/3/001

10. Gerth, W. and C. Watke, Electrical Impedance Spectroscopic Monitoring of Body Compartmental Volume Changes. Clinical Medicine, 1993: p. 8.

11. Dantchev, S. and F. Al-Hatib, Nonlinear curve fitting for bioelectrical impedance data analysis: a minimum ellipsoid volume method. Physiol Meas, 1999. 20(1): p. N1-9. doi: 10.1088/0967-3334/20/1/009

12. Kun, S., et al., Real-time extraction of tissue impedance model parameters for electrical impedance spectrometer. Medical \& Biological Engineering \& Computing, 1999. 37(4): p. 428-432. doi: 10.1007/BF02513325

13. Ward, L.C., T. Essex, and B.H. Cornish, Determination of Cole parameters in multiple frequency bioelectrical impedance analysis using only the measurement of impedances. Physiol Meas, 2006. 27(9): p. 839-50. doi: 10.1088/0967-3334/27/9/007

14. Ayllón, D., F. Seoane, and R. Gil-Pita, Cole Equation and Parameter Estimation from Electrical Bioimpedance Spectroscopy Measurements - A Comparative Study, in EMBC 2009. 2009, IEEE: Minneapolis. p. 3779-3782.
15. Buendia, R., R. Gil-Pita, and F. Seoane, Experimental validation of a method for removing the capacitive leakage artifact from electrical bioimpedance spectroscopy measurements. Measurements, Science and Technology, 2010. 21(11): p. 8. doi:10.1088/1742-6596/224/1/012126

16. Nordbotten, B.J., et al., Evaluation of algorithms for calculating bioimpedance phase angle values from measured whole-body impedance modulus. Physiol Meas., 2011. 32(7): p. 755-65. Epub 2011 Jun 7. doi: $10.1088 / 0967-3334 / 32 / 7 / \mathrm{S} 03$

17. De Lorenzo, A., et al., Predicting body cell mass with bioimpedance by using theoretical methods: a technological review. J Appl Physiol, 1997. 82(5): p. 1542-58.

18. Van Loan, M.D., et al., Fluid changes during pregnancy: use of bioimpedance spectroscopy. J Appl Physiol, 1995. 78(3): p. $1037-42$.

19. Van Loan, et al., Use of bio-impedance spectroscopy (BIS) to determine extracellular fluid (ECF), intracellular fluid (ICF), total body water (TBW), and fat-free mass (FFM). . Human Body Composition In Vivo: Methods, Models, and Assessment, , 1993: p. 4.

20. Scharfetter, H., et al., A model of artefacts produced by stray capacitance during whole body or segmental bioimpedance spectroscopy. Physiological Measurement, 1998. 19(2): p. 247-261. doi: 10.1088/0967-3334/19/2/012 